\title{
AIM2 inhibits the proliferation, invasion and migration, and promotes the apoptosis of osteosarcoma cells by inactivating the PI3K/AKT/mTOR signaling pathway
}

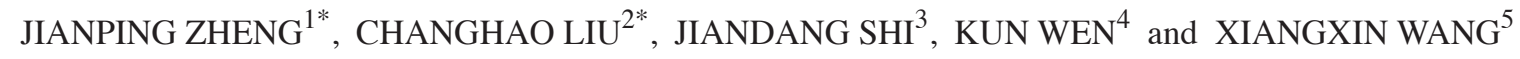 \\ ${ }^{1}$ Department of Orthopedic Traumatology, General Hospital of Ningxia Medical University, Yinchuan, \\ Ningxia Hui Autonomous Region 750004; ${ }^{2}$ Department of Orthopedics, Zhangye People's Hospital Affiliated to \\ Hexi University, Zhangye, Gansu 734000; ${ }^{3}$ Department of Spine Surgery, General Hospital of Ningxia Medical University, \\ Yinchuan, Ningxia Hui Autonomous Region 750004; ${ }^{4}$ Department of Orthopedics, Ningxia Gemflower Hospital, \\ Yinchuan, Ningxia Hui Autonomous Region 750006; ${ }^{5}$ Ningxia Medical University, Yinchuan, \\ Ningxia Hui Autonomous Region 750001, P.R. China
}

Received August 12, 2021; Accepted November 17, 2021

DOI: $10.3892 / \mathrm{mmr} .2021 .12569$

\begin{abstract}
Osteosarcoma is a primary bone tumor that mainly occurs in children and adolescents. Absent in melanoma 2 (AIM2) has been demonstrated to be involved in regulating the occurrence and development of cancer, exerting oncogenic and pro-cancer effects; however, its role in osteosarcoma is poorly understood. The present study aimed to explore the function and molecular mechanism of AIM2 in the progression of osteosarcoma. In the present study, AIM2 expression was predicted using the Cancer Cell Line Encyclopedia database and examined in several osteosarcoma cell lines using reverse transcription-quantitative PCR and western blotting. Following AIM2 overexpression, cell proliferation and apoptosis were examined using Cell Counting Kit-8, colony formation and TUNEL staining assays. The expression levels of proteins related to apoptosis, epithelial-mesenchymal transition (EMT) and the PI3K/AKT/mTOR signaling pathway were determined by western blotting. Additionally, cell invasion and migration were assessed using Transwell and wound healing assays. After addition of the PI3K/AKT/mTOR signaling pathway inhibitor LY294002 or activator 740Y-P, cell function analysis was performed. The results demonstrated that AIM2 was expressed at low levels in osteosarcoma cell lines. AIM2 overexpression inhibited proliferation, invasion, migration and EMT,
\end{abstract}

Correspondence to: Dr Jiandang Shi, Department of Spine Surgery, General Hospital of Ningxia Medical University, 804 Shengli South Street, Xingqing, Yinchuan, Ningxia Hui Autonomous Region 750004, P.R. China

E-mail: shijiandang101@163.com

\section{*Contributed equally}

Key words: absent in melanoma 2, osteosarcoma, PI3K/AKT/mTOR, epithelial-mesenchymal transition, 740Y-P and promoted apoptosis in osteosarcoma cells. Furthermore, the levels of phosphorylated (p)-PI3K, p-AKT and p-mTOR were markedly downregulated following AIM2 overexpression. Furthermore, LY294002 treatment had the same effects as AIM2 upregulation on osteosarcoma cell proliferation, apoptosis, invasion, migration and EMT. By contrast, 740Y-P reversed the effects of AIM2 overexpression on the behavior of osteosarcoma cells. Overall, the findings of the present study demonstrated that AIM2 may inhibit the progression of osteosarcoma by inactivating the PI3K/AKT/mTOR signaling pathway, and suggested that AIM2 may be a promising marker for the treatment of osteosarcoma.

\section{Introduction}

Osteosarcoma is a malignant tumor caused by osteoblastic mesenchymal cells, mostly occurring in children and young adults (1). The primary site of growth of osteosarcoma is the epiphyseal region of long bones (2). Although novel technologies have enabled early diagnosis of osteosarcoma, and improvements in surgery and chemotherapy have increased the 5-year survival rate for osteosarcoma from $<20 \%$ to $65-75 \%$ (3), the local recurrence rate is $\sim 10 \%$, and the survival rate of patients with metastases or relapses is only $10 \%$ (4). Currently, tumor metastasis is the primary issue in osteosarcoma treatment (5). Since $80 \%$ of patients with osteosarcoma already exhibit signs of metastasis or micro-metastasis at the time of diagnosis, almost all patients undergo multiagent chemotherapy in addition to surgical treatment (6). Accordingly, understanding the mechanisms of tumor development and finding reliable biomarkers and therapeutic targets is crucial for the treatment of osteosarcoma.

Absent in melanoma 2 (AIM2) is a cytosolic innate immune receptor with the ability to recognize double-stranded DNA (dsDNA) (7). AIM2 not only promotes the development of sterile inflammatory diseases, such as chronic kidney disease (8) and cardiovascular diseases (9), but also participates in regulating the occurrence and development of certain 
cancers, serving a dual role of cancer inhibition and promotion. For instance, data from several studies suggest that AIM2 expression is reduced in malignant tumors, such as colon cancer (10), liver cancer (11), prostate cancer (12) and breast cancer (13), thereby promoting tumorigenesis and affecting patient prognosis. Conversely, it has previously been observed that AIM2 expression is upregulated in oral squamous carcinoma (14), squamous carcinoma (15) and lung cancer (16). These studies also revealed that knockdown of AIM2 inhibits tumor cell proliferation, promotes apoptosis and reduces cell migration, indicating that AIM2 functions as an oncogene in oral squamous carcinoma (14), squamous carcinoma (15) and lung cancer (16). However, little attention has been paid to the role served by AIM2 in osteosarcoma and its molecular mechanisms.

The PI3K/AKT/mTOR signaling pathway has been demonstrated to be one of the most common abnormalities in the metastatic behavior of osteosarcoma, as activation of this signaling pathway speeds up cell metastasis and is associated with adverse prognosis in osteosarcoma (17). A previous study revealed that AIM2 can regulate the development of colorectal cancer via the PI3K/AKT signaling pathway (18), which has later been demonstrated to play a key role in the progression of osteosarcoma (19). Furthermore, evidence has suggested that AIM2 expression deficiency promotes hepatocellular carcinoma progression by activating the mTOR-ribosomal protein S6 kinase B1 signaling pathway (11). Therefore, it was hypothesized that AIM2 may regulate the development of osteosarcoma via the PI3K/AKT/mTOR signaling pathway.

In the present study, the expression levels of AIM2 in several osteosarcoma cell lines were examined. Subsequently, the effects of AIM2 overexpression on the proliferation, apoptosis, invasion, migration and epithelial-mesenchymal transition (EMT) of osteosarcoma cells, as well as potential mechanisms related to the PI3K/AKT/mTOR signaling pathway, were explored.

\section{Materials and methods}

Cell culture and transfection. hFOB1.19 human osteoblast cells were obtained from American Type Culture Collection and cultured in DMEM/F12 (Invitrogen; Thermo Fisher Scientific, Inc.) supplemented with 10\% FBS (Sangon Biotech Co., Ltd.) and $1 \%$ penicillin-streptomycin. C396, CAL-72 and MG-63 osteosarcoma cell lines were purchased from The Cell Bank of Type Culture Collection of The Chinese Academy of Sciences, and cultured in DMEM supplemented with $10 \%$ FBS and $1 \%$ penicillin-streptomycin. All cells were cultured at $37^{\circ} \mathrm{C}$ in a suitable incubator containing $95 \%$ air and $5 \% \mathrm{CO}_{2}$. MG-63 cells were treated with PI3K/AKT/mTOR signaling pathway inhibitor LY294002 (20 $\mu \mathrm{M}$; cat. no. A8250; APExBIO Technology LLC) or activator 740Y-P (20 $\mu \mathrm{M}$; cat. no. BCP16353; Shanghai BioChemPartner Co., Ltd.) at $24 \mathrm{~h}$ post-transfection for $48 \mathrm{~h}$ at $37^{\circ} \mathrm{C}$.

For transfection, AIM2 overexpression plasmid (Oe-AIM2) and the empty vector plasmid (Oe-NC) were purchased from Sangon Biotech Co., Ltd., by utilizing pcDNA3.1 vector containing the full-length AIM2 cDNA sequence. Subsequently, these plasmids were transfected into MG-63 cells using Lipofectamine ${ }^{\circledR} 2000$ transfection reagent
(Invitrogen; Thermo Fisher Scientific, Inc.) according to the manufacturer's instructions. After $48 \mathrm{~h}$ of transfection, cells were collected and the transfection efficiency was assessed via reverse transcription-quantitative PCR (RT-qPCR) and western blotting, and subsequent experiments were performed.

$R T-q P C R$ assay. Extraction of total RNA from cells was performed using TRIzol ${ }^{\circledR}$ reagent (Thermo Fisher Scientific, Inc.) according to the manufacturer's instructions. The cDNA template in the reverse transcription reaction was generated using a QuantiTect Reverse Transcription Kit provided by Qiagen China Co., Ltd., according to the manufacturer's protocols. The gene expression levels were analyzed via qPCR, which was conducted using iTaq $^{\mathrm{TM}}$ Universal One-Step iTaq ${ }^{\mathrm{TM}}$ Universal SYBR ${ }^{\circledR}$-Green Supermix (Bio-Rad Laboratories, Inc.) on an ABI 7500 instrument (Applied Biosystems; Thermo Fisher Scientific, Inc.). The following primer sequences were used for qPCR: AIM2 forward, 5'-TGGCAAAACGTCTTC AGGAGG-3' and reverse, 5'-AGCTTGACTTAGTGGCTT TGG-3'; GAPDH forward, 5'-GGAGCGAGATCCCTCCAA AAT-3' and reverse, 5'-GGCTGTTGTCATACTTCTCAT GG-3'. The quantification of relative gene expression was performed using the $2^{-\Delta \Delta \mathrm{Cq}}$ method (20). GAPDH was used as an internal control for normalization.

Western blotting. Cell lysis was performed on ice using RIPA buffer (Beyotime Institute of Biotechnology) to obtain proteins. The protein concentration was measured using a BCA protein assay kit (GlpBio Technology, Inc.). The separation of proteins (30 $\mu \mathrm{g}$ per lane) was accomplished using $10 \%$ SDS-PAGE. Subsequently, the separated proteins were transferred onto polyvinylidene fluoride membranes. After $1 \mathrm{~h}$ of blocking with $5 \%$ skimmed milk at $37^{\circ} \mathrm{C}$, the membranes and primary antibodies were incubated together at $4^{\circ} \mathrm{C}$ overnight. After three washes, the membranes were incubated with a horseradish peroxidase-linked secondary antibody (1:3,000; cat. no. 7074P2; Cell Signaling Technology, Inc.) at room temperature. Pierce ${ }^{\mathrm{TM}}$ enhanced chemiluminescence western blotting substrate (Pierce; Thermo Fisher Scientific, Inc.) was used for visualization, according to the manufacturer's instructions, and ImageJ software (v6; National Institutes of Health) was used to analyze the protein bands. Parallel blotting of GAPDH was used as the internal control. Anti-AIM2 (1:1,000; cat. no. 12948S), anti-Bax (1:1,000; cat. no. 5023T), anti-Bcl-2 (1:1,000; cat. no. 3498T), anti-cleaved caspase-3 (1:1,000; cat. no. 9664T), caspase-3 (1:1,000; cat. no. $14220 \mathrm{~T})$, anti-N-cadherin (1:1,000; cat. no. 13116T), anti-Vimentin (1:1,000; cat. no. 5741T), anti-E-cadherin (1:1,000; cat. no. 3195T), anti-phosphorylated (p)-AKT (1:1,000; cat. no. 4060T), anti-AKT (1:1,000; cat. no. 4691T), anti-p-mTOR (1:1,000; cat. no. 5536T), anti-mTOR (1:1,000; cat. no. 2983T) and anti-GAPDH (1:1,000; cat. no. 5174T) antibodies were provided by Cell Signaling Technology, Inc. Anti-p-PI3K (1:1,000; cat. no. ab278545) and anti-PI3K (1:1,000; cat. no. ab32089) antibodies were obtained from Abcam.

Cell Counting Kit-8 (CCK-8) assay. For the cell viability assay, MG-63 cells were plated into 96-well plates and cultured at $37^{\circ} \mathrm{C}$ for $24 \mathrm{~h}$. Subsequently, $10 \mu \mathrm{l} \mathrm{CCK}-8$ solution 
(MedChemExpress) was loaded into each well and the cells were incubated for $3 \mathrm{~h}$ at $37^{\circ} \mathrm{C}$. Optical density was measured using a microplate reader at a wavelength of $450 \mathrm{~nm}$. Each group of experiments was repeated three times.

Colony formation assay. For the detection of colony formation, MG-63 cells in the logarithmic growth phase were cultured and made into cell suspensions under specified conditions. The cell suspensions were diluted and inoculated into 60 -mm diameter culture dishes at a density of 500 cells per dish, which were gently shaken in a cross direction to ensure the cells were dispersed evenly. The culture dishes were placed at $37^{\circ} \mathrm{C}$ in a $5 \% \mathrm{CO}_{2}$ environment for 2 weeks. Then, the culture was terminated and the culture medium was discarded. After washing with PBS twice, cells were fixed with methanol at room temperature for $15 \mathrm{~min}$ and stained with crystal violet (Beijing Solarbio Science \& Technology Co., Ltd.) staining solution at room temperature for $10 \mathrm{~min}$. The staining solution was subsequently washed away. Finally, each colony of $>30$ cells was counted using a dissection microscope.

Detection of apoptosis. The apoptosis of MG-63 cells was assessed using a TUNEL assay (Beyotime Institute of Biotechnology) according to the manufacturer's instructions. After $24 \mathrm{~h}$ of cell culture, transfected cells were fixed with $1 \%$ paraformaldehyde at room temperature for $15 \mathrm{~min}$ and $50 \mu 1$ TUNEL was used to incubate cells for $1 \mathrm{~h}$ at $37^{\circ} \mathrm{C}$, followed by staining of nuclear DNA with $10 \mu \mathrm{g} / \mathrm{ml}$ DAPI at $37^{\circ} \mathrm{C}$ for 2-3 min and mounted in an anti-fade reagent (Beijing Solarbio Science \& Technology Co., Ltd.). The cells were analyzed from three fields of view using a fluorescence microscope (magnification, x200; Olympus Corporation).

Transwell assay. The upper surface of the Transwell chamber was coated with Matrigel and incubated at $37^{\circ} \mathrm{C}$ for $30 \mathrm{~min}$.

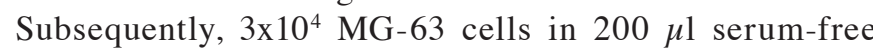
medium were seeded onto the upper surface of the chamber. A total of $600 \mu \mathrm{l}$ medium containing $10 \%$ FBS was added to the lower chamber. After incubation at room temperature for $24 \mathrm{~h}$, cells were fixed with $4 \%$ paraformaldehyde for $20 \mathrm{~min}$ at $4^{\circ} \mathrm{C}$ and stained with $0.1 \%$ crystal violet solution (Beijing Solarbio Science \& Technology Co., Ltd.) for $5 \mathrm{~min}$ at room temperature. Finally, these cells were observed under an inverted microscope. Invasive cells were counted in three random areas under a light microscope (magnification, x100; Olympus Corporation).

Wound healing assay. For the evaluation of cell migration, MG-63 cells $\left(4 \times 10^{5}\right.$ cells/well) were seeded into six-well plates and cultured for $48 \mathrm{~h}$. A $20-\mu 1$ pipette tip was utilized to create a straight scratch and the medium was replaced with serum-free DMEM. After two washes with PBS, the wound was observed at 0 and $24 \mathrm{~h}$. The microscopy images of the cells were captured using a light microscope (magnification, x100; Olympus Corporation). The cell migration rate was calculated using the following equation: (Initial width at $0 \mathrm{~h}$-final width at $24 \mathrm{~h}$ )/Initial width at $0 \mathrm{~h}$.

Statistical analysis. Experimental data are presented as the mean \pm SD from three independent experiments and were analyzed using GraphPad Prism 8.0 software (GraphPad Software, Inc.). The normality of the data was analyzed using the Shapiro-Wilk test. One-way ANOVA followed by Tukey's post hoc test and unpaired t-tests were used for comparisons among multiple groups and between two groups, respectively. $\mathrm{P}<0.05$ was considered to indicate a statistically significant difference.

Bioinformatics tools. Cancer Cell Line Encyclopedia (CCLE) database (https://depmap.org/portal/download/?release=CCLE+ 2019\&release $=$ Fusion $\&$ release $=\mathrm{DNA}+\mathrm{Copy}+$ Number; dataset: 'CCLE_Expression.Arrays_2013-03-18.tar.gz') was applied to investigate AIM2 expression in osteosarcoma.

\section{Results}

AIM2 is expressed at low levels in osteosarcoma cell lines. The CCLE database, RT-qPCR and western blotting were used to gain a deeper insight into AIM2 expression in osteosarcoma cell lines. As shown in Fig. 1A, AIM2 was expressed at low levels in osteosarcoma cell lines, according to the CCLE database. The results in Fig. $1 \mathrm{~B}$ and $\mathrm{C}$ indicated that both mRNA and protein expression levels of AIM2 were significantly decreased in C396, CAL-72 and MG-63 cells compared with hFOB1.19 cells. Among these, MG-63 cells were selected for the subsequent experiments due to their low expression levels of AIM2. Overall, the results suggested that AIM2 expression was downregulated in osteosarcoma cells.

Overexpression of AIM2 inhibits cell proliferation and promotes apoptosis in osteosarcoma. To gain a detailed understanding of the effect of AIM2 on cell proliferation and apoptosis in osteosarcoma, MG-63 cells were transfected with an AIM2 overexpression plasmid, followed by a series of experiments. First, data from RT-qPCR and western blotting indicated a significant increase in AIM2 expression in the overexpression group (Fig. 2A and B) compared with the empty vector group. AIM2 overexpression significantly inhibited the proliferation of osteosarcoma cells according to the CCK-8 and colony formation assays (Fig. 2C and D). As shown in Fig. 2E, AIM2 upregulation led to an increase in the number of apoptotic cells compared with the empty vector group. As indicated in Fig. 2F, the results revealed elevated expression levels of apoptosis-related proteins Bax and cleaved caspase-3, as well as decreased expression levels of Bcl-2, following AIM2 overexpression. In summary, AIM2 overexpression effectively suppressed proliferation and promoted apoptosis in osteosarcoma cells.

Overexpression of AIM2 suppresses the invasion, migration and EMT of osteosarcoma cells. To explore the effect of AIM2 overexpression on the invasion, migration and EMT of osteosarcoma cells, Transwell, wound healing and western blotting assays were performed. After AIM2 was overexpressed, a significant decrease in cell invasion compared with the Oe-NC group was observed (Fig. 3A). The results in Fig. 3B also revealed a significant decrease in the migration of MG-63 cells. Additionally, the results in Fig. 3C revealed a significant decrease in the expression levels of the EMT-related proteins $\mathrm{N}$-cadherin and vimentin, but an increase in the expression 

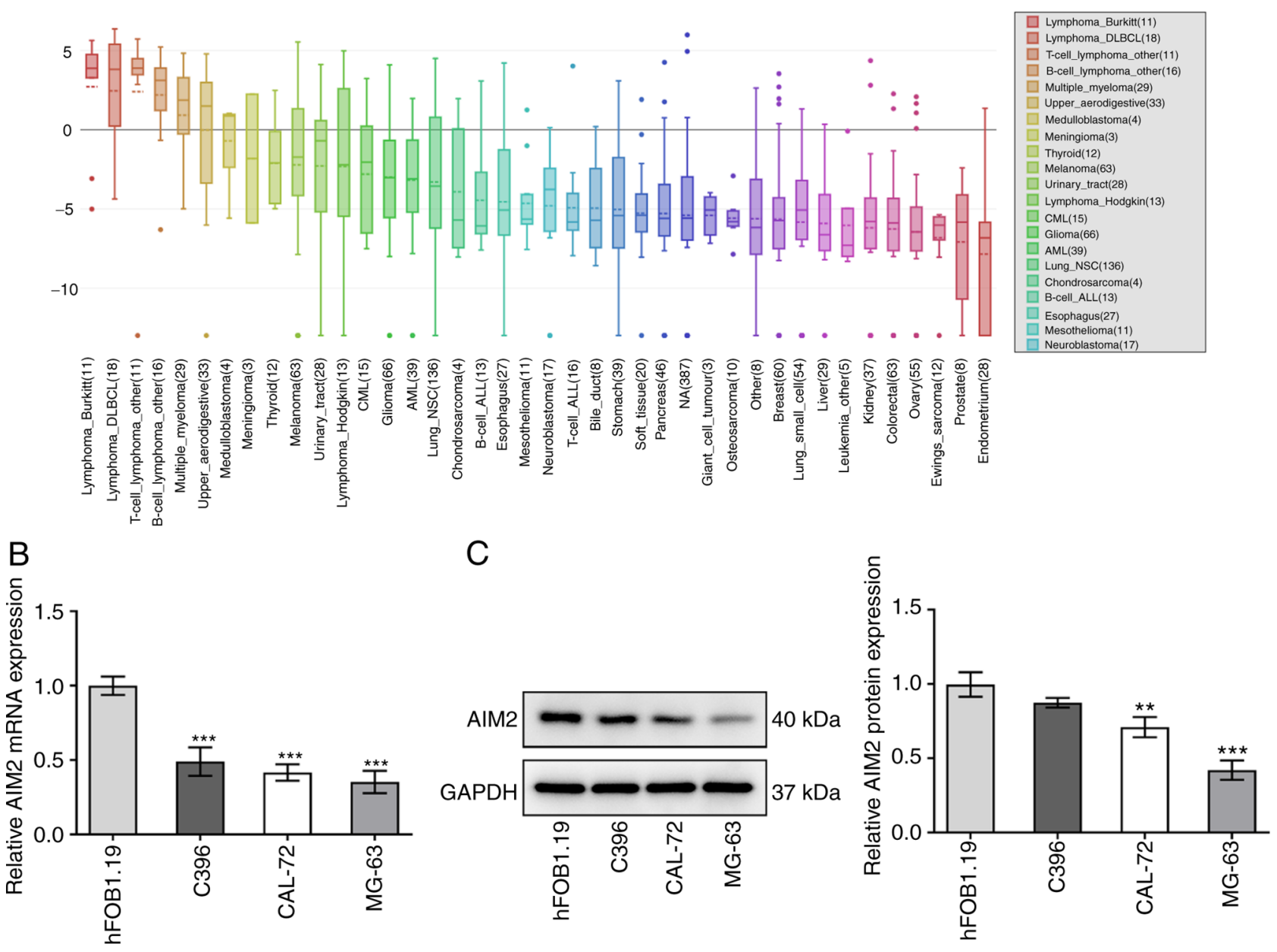

Figure 1. AIM2 is expressed at low levels in osteosarcoma cell lines. (A) Cancer Cell Line Encyclopedia database indicated AIM2 expression in various cancer type cell lines, including osteosarcoma. Detection of AIM2 expression in osteosarcoma cell lines was performed via (B) reverse transcription-quantitative PCR and (C) western blotting. Experimental data are presented as the mean \pm SD from three independent experiments. ${ }^{* * *} \mathrm{P}<0.01,{ }^{* * * *} \mathrm{P}<0.001 \mathrm{vs}$. $\mathrm{hFOB} 1.19$. AIM2, absent in melanoma 2.

levels of E-cadherin, indicating that the EMT process was inhibited following AIM2 overexpression. These data suggested that AIM2 overexpression exerted an inhibitory effect on the invasion, migration and EMT of osteosarcoma cells.

AIM2 overexpression suppresses the PI3K/AKT/mTOR signaling pathway in osteosarcoma cells. The present study subsequently examined if AIM2 overexpression affected the $\mathrm{PI} 3 \mathrm{~K} / \mathrm{AKT} / \mathrm{mTOR}$ signaling pathway following treatment with the inhibitor LY294002 or the activator 740Y-P. Decreased protein levels of $\mathrm{p}-\mathrm{P} 13 \mathrm{~K}, \mathrm{p}-\mathrm{AKT}$ and $\mathrm{p}-\mathrm{mTOR}$ relative to PI3K, AKT and mTOR were observed in the Oe-AIM2 group compared with the Oe-NC group (Fig. 4A). In MG-63 cells transfected with Oe-NC, after the addition of inhibitor LY294002, the levels of p-P13K, p-AKT and p-mTOR were also significantly downregulated compared with those in the Oe-NC group (Fig. 4B). By contrast, a significant increase in the levels of p-P13K, p-AKT and p-mTOR was observed following addition of 740Y-P in MG-63 cells overexpressing AIM2. In summary, these results indicated that AIM2 overexpression could suppress the PI3K/AKT/mTOR signaling pathway in osteosarcoma cells.
AIM2 overexpression inhibits the proliferation and promotes the apoptosis of osteosarcoma cells by inactivating the PI3K/AKT/mTOR signaling pathway. To understand whether the effects of AIM2 overexpression on the progression of osteosarcoma were mediated by the PI3K/AKT/mTOR signaling pathway, another series of experiment was conducted. The results in Fig. 5A and B demonstrated that the inhibitor LY294002 exerted a significant inhibitory effect on cell proliferation in both the CCK-8 and colony formation assays, as did AIM2 overexpression, while the activator 740Y-P reversed the inhibitory effect of AIM2 overexpression. As shown in Fig. 5C, similar to the effect of AIM2 overexpression, the inhibitor LY294002 promoted apoptosis, but 740Y-P attenuated the effect of AIM2 overexpression on cell apoptosis. As shown in Fig. 5D, following addition of LY294002, the protein expression levels of Bax and cleaved caspase-3 were elevated, but the expression levels of Bcl-2 were decreased compared with Oe-NC group, and treatment with the activator 740Y-P had the opposing effect on the levels of these proteins in Oe-AIM2 cells. These results suggested that AIM2 inhibited the proliferation and promoted the apoptosis of osteosarcoma cells by inactivating the PI3K/AKT/mTOR signaling pathway. 


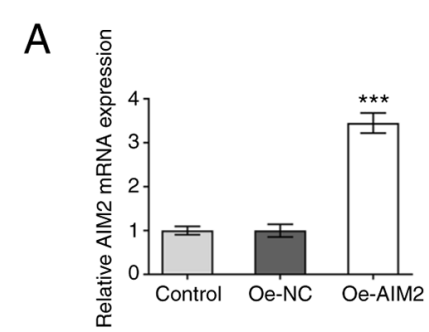

D

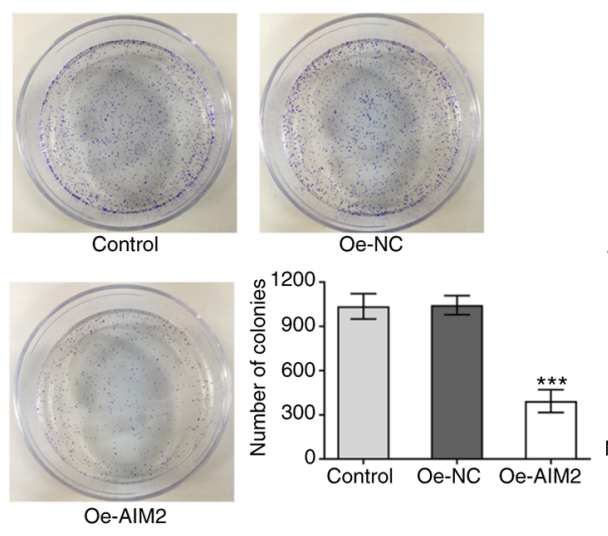

F

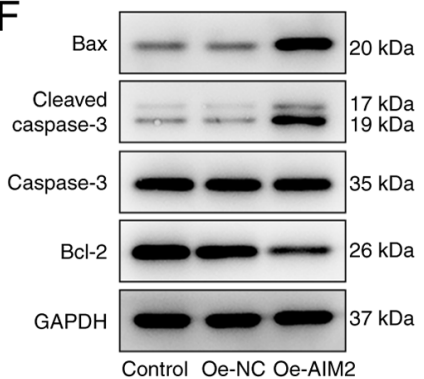

B

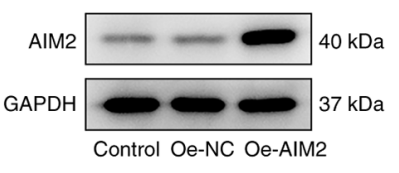

E

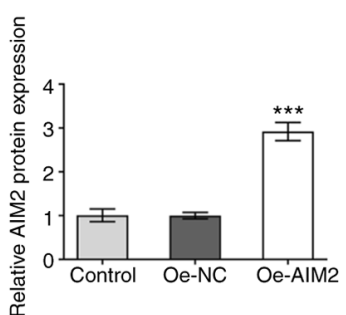

C

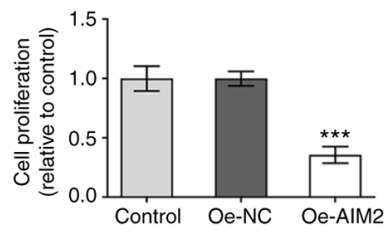

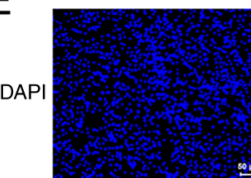
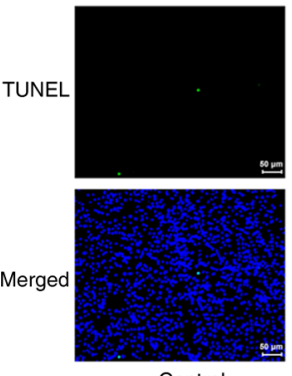

Control
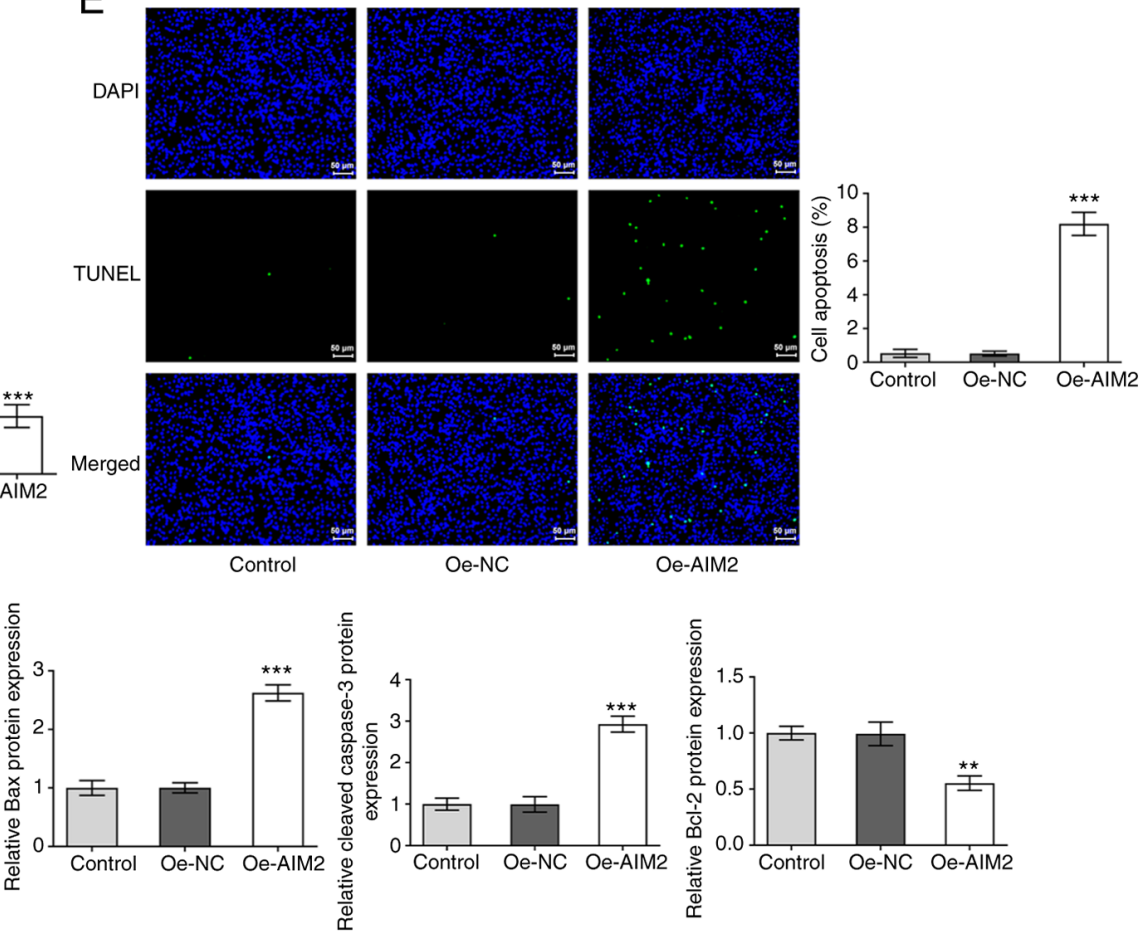

Oe-NC

Oe-AIM2
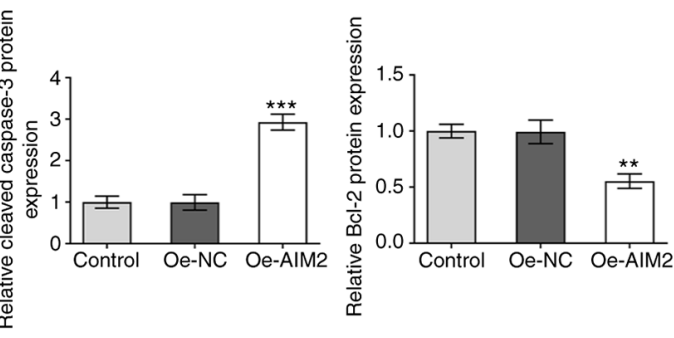

Figure 2. Overexpression of AIM2 inhibits proliferation and promotes apoptosis in osteosarcoma cells. (A) Reverse transcription-quantitative PCR and (B) western blotting were used to detect the transfection efficiency of overexpression of AIM2. (C) Cell Counting Kit-8 and (D) colony formation assays were implemented to examine the effect of AIM2 on the proliferation of osteosarcoma cells. (E) Apoptosis of osteosarcoma cells was detected using TUNEL staining. Scale bar, $50 \mu \mathrm{m}$. (F) Expression levels of apoptosis-related proteins Bax, cleaved caspase-3 and Bcl-2 were determined via western blotting. Experimental data are presented as the mean $\pm \mathrm{SD}$ from three independent experiments. ${ }^{* *} \mathrm{P}<0.01,{ }^{* * * *} \mathrm{P}<0.001$ vs. Oe-NC. AIM2, absent in melanoma 2 ; Oe-NC, empty vector plasmid; Oe-AIM2, AIM2 overexpression vector.

AIM2 overexpression inhibits the invasion, migration and EMT of osteosarcoma cells by inactivating the PI3K/AKT/mTOR signaling pathway. In terms of cell invasion, the results shown in Fig. 6A revealed that LY294002 effectively suppressed cell invasion compared with the Oe-NC group; invasion was significantly elevated following addition of 740Y-P in MG-63 cells transfected with Oe-AIM2. As shown in Fig. 6B, LY294002 had the same inhibitory effect as AIM2 overexpression on cell migration, while 740Y-P had the opposite effect. Based on the results in Fig. 6C, the expression levels of EMT-related proteins $\mathrm{N}$-cadherin and vimentin were decreased but E-cadherin expression was increased following addition of LY294002 compared with Oe-NC alone. Following overexpression of AIM2 and addition of the activator $740 \mathrm{Y}-\mathrm{P}$, the expression levels of $\mathrm{N}$-cadherin and vimentin were found to be increased, but the expression levels of E-cadherin were decreased. This series of experiments indicated that the inhibitor LY294002 suppressed cell invasion, migration and EMT, but the activator 740Y-P reversed the effect of AIM2 overexpression on the invasion, migration and EMT of osteosarcoma cells.

\section{Discussion}

Osteosarcoma is a primary bone malignant tumor with a high recurrence rate. Although a growing body of research has demonstrated an association between AIM2 and the progression of cancer $(18,21,22)$, research into the role of AIM2 in osteosarcoma has been limited. The present study assessed the role of AIM2 in osteosarcoma cells and its molecular mechanism, and demonstrated that AIM2 was expressed at low levels in osteosarcoma cell lines. Additionally, overexpression of AIM2 inhibited the proliferation, invasion, migration and EMT, and promoted the apoptosis of osteosarcoma cells by suppressing the PI3K/AKT/mTOR signaling pathway.

AIM2 is the prototypical and most well-characterized member of the AIM2 class of receptors (23). Recognition of dsDNA by AIM2 results in the accumulation of a large multiprotein oligomeric complex referred to as the inflammasome, which gives rise to the secretion of bioactive IL-1 $\beta$ and IL-18 (21). In the inflammatory microenvironment of tumors, caspase-1 and the cytokines it processes, such as IL-1 $\beta$, serve an essential role in the occurrence and 
A

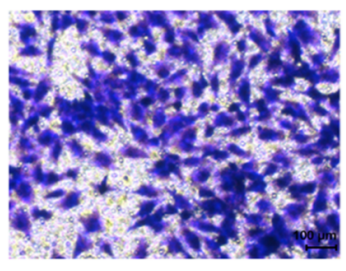

B
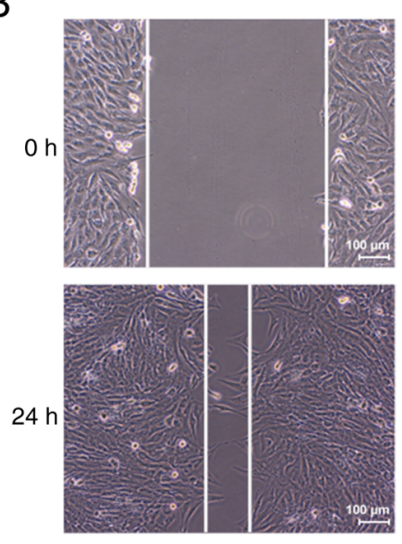

Control

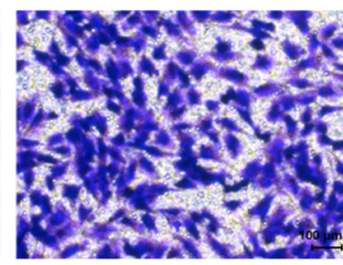

Oe-NC
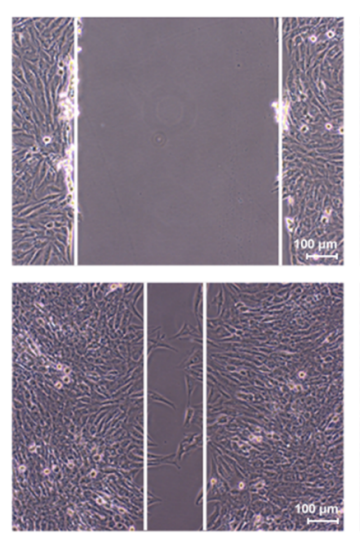

Oe-NC

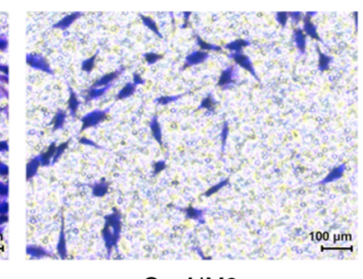

Oe-AIM2

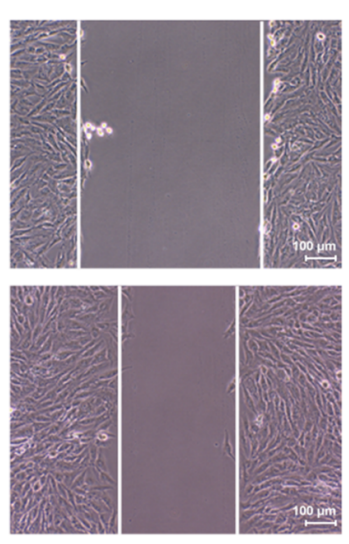

Oe-AIM2
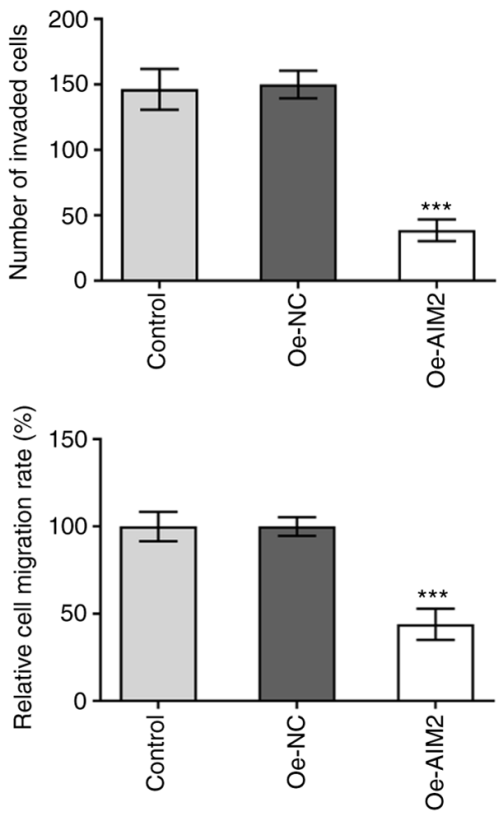

C
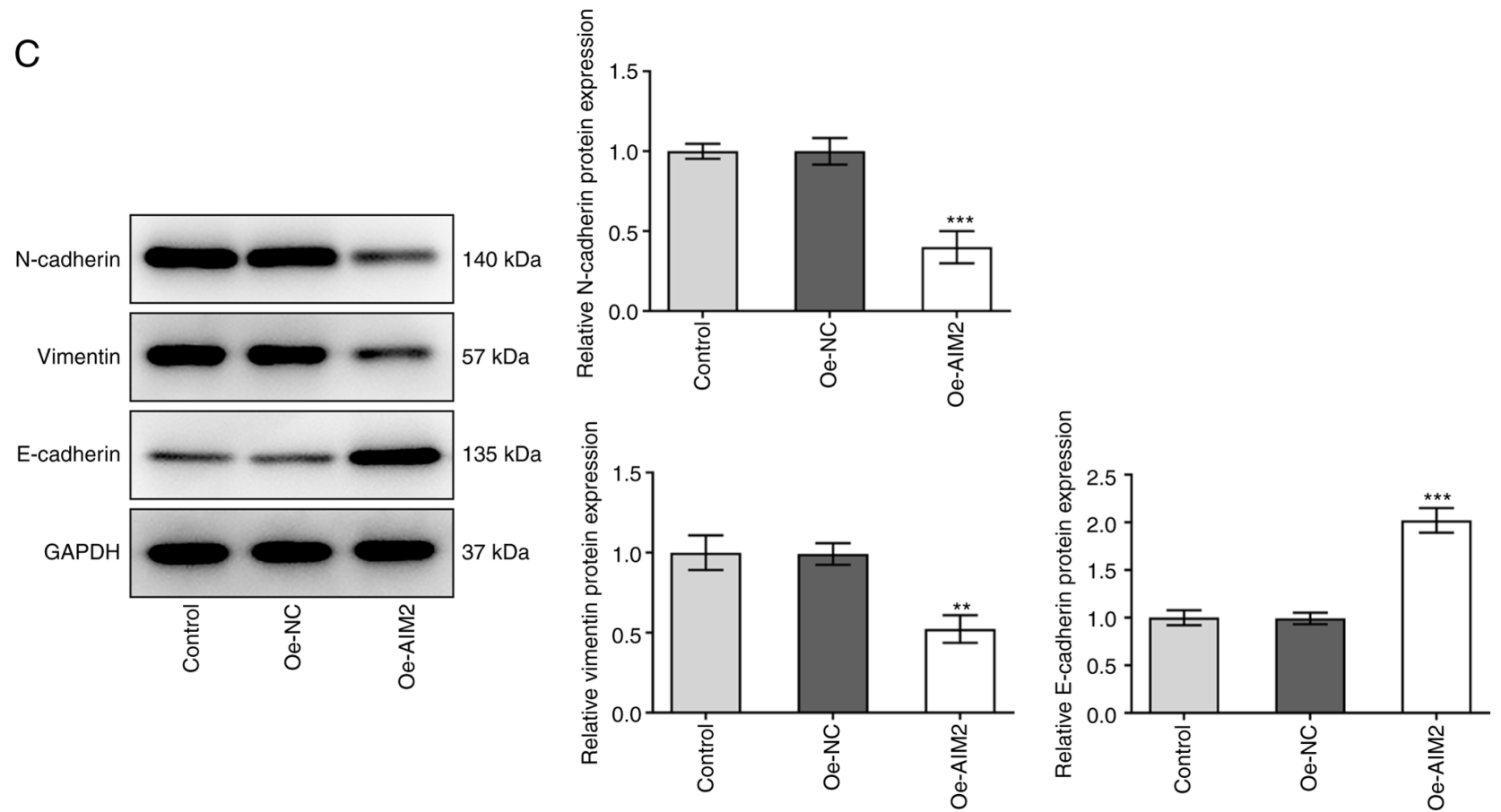

Figure 3. Overexpression of AIM2 inhibits osteosarcoma cell invasion, migration and EMT. (A) Cell invasion was detected using a Transwell assay. Scale bar, $100 \mu \mathrm{m}$. (B) Wound healing assay was utilized to detect cell migration. Scale bar, $100 \mu \mathrm{m}$. (C) Western blotting was used to assess the levels of EMT-related proteins, including $\mathrm{N}$-cadherin, Vimentin and E-cadherin. Experimental data are presented as the mean \pm SD from three independent experiments. ${ }^{* *} \mathrm{P}<0.01,{ }^{* * * * *} \mathrm{P}<0.001$ vs. Oe-NC. EMT, epithelial-mesenchymal transition; AIM2, absent in melanoma 2; Oe-NC, empty vector plasmid; Oe-AIM2, AIM2 overexpression vector.

development of cancer (24). Also, caspase-1 functions as a tumor suppressor in breast cancer (25) and non-small cell lung cancer (26). Additionally, the CCLE database revealed that AIM2 expression was downregulated in osteosarcoma cell lines, which was also validated by RT-qPCR and western blot assays in the present study. It may be inferred from these results that AIM2 may contribute to the pathogenesis of osteosarcoma.

The proliferation, migration and invasion of tumor cells mark the metastasis of tumors, which in turn causes cancer recurrence in patients $(27,28)$. AIM2 has been demonstrated to be closely associated with the progression of cancer in recent studies $(16,18)$. It has been reported that overexpression of AIM2 induces cell cycle arrest in colon cancer cytology and acts as an inhibitor of cell proliferation (29). Furthermore, in organoid cultures, intestinal stem cells lacking AIM2 proliferate more than wild-type intestinal stem cells (30). In addition, AIM2 regulates the viability and apoptosis of human colorectal cancer cells via the PI3K/AKT signaling pathway (31). It has been demonstrated that, in 

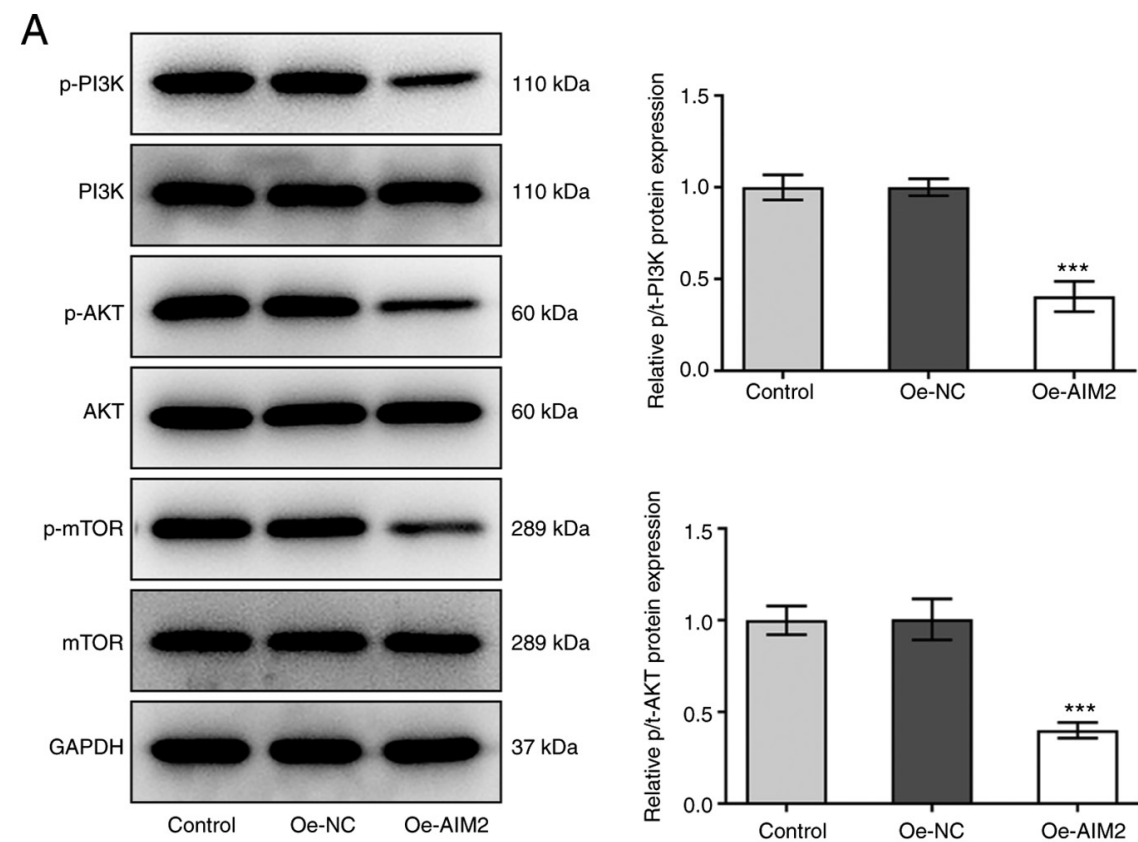

B
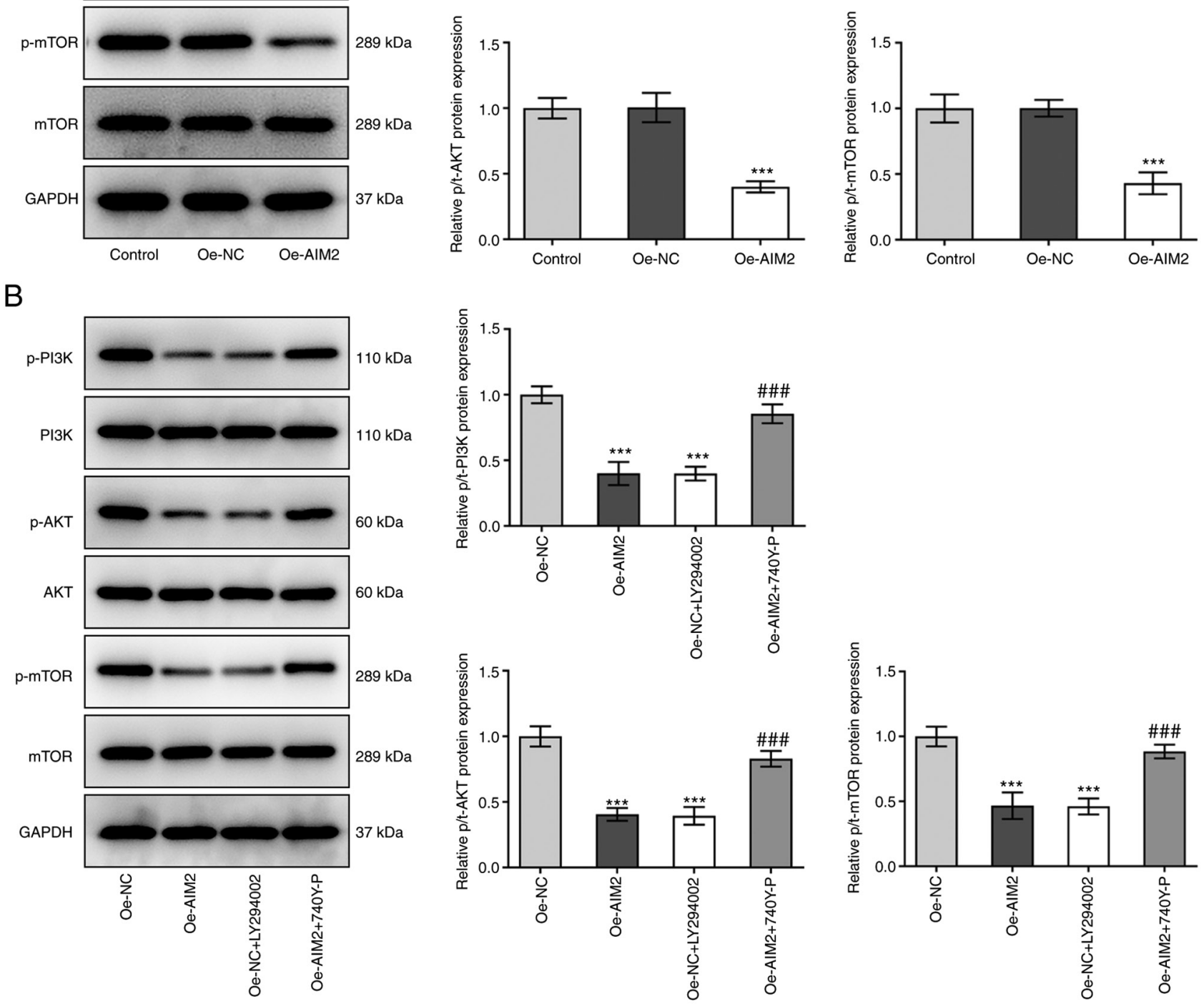

Figure 4. AIM2 suppresses the PI3K/AKT/mTOR signaling pathway. (A) Expression levels of PI3K/AKT/mTOR signaling pathway-related proteins were measured by western blotting after AIM2 overexpression. (B) Detection of PI3K/AKT/mTOR pathway-related protein expression was performed by western

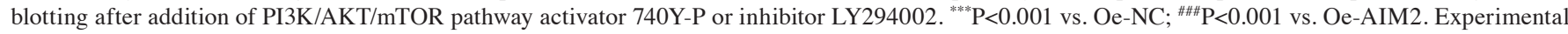
data are presented as the mean \pm SD from three independent experiments. AIM2, absent in melanoma 2; Oe-NC, empty vector plasmid; Oe-AIM2, AIM2 overexpression vector; $\mathrm{p}$, phosphorylated; $\mathrm{t}$, total.

AIM2 knockout mice, the production of pro-inflammatory cytokines mediated by the inflammasome is reduced, and apoptosis and pyroptosis are attenuated (32). Furthermore, AIM2 inhibits the invasion and metastasis of kidney cancer cells by enhancing autophagy induction (33). AIM2 overexpression inhibits colorectal cancer cell proliferation, migration and EMT progress (18). Furthermore, the loss of AIM2 induces the activation of EMT in hepatocellular carcinoma (34). Based on the results of the present study, overexpression of AIM2 inhibited osteosarcoma cell proliferation, invasion, migration and EMT, and promoted apoptosis, which indicated that AIM2 can suppress the progression of osteosarcoma.

The PI3K/AKT/mTOR signaling pathway serves a critical role in the regulation of cell proliferation, survival, protein synthesis and tumor progression $(35,36)$. PI3K 
A
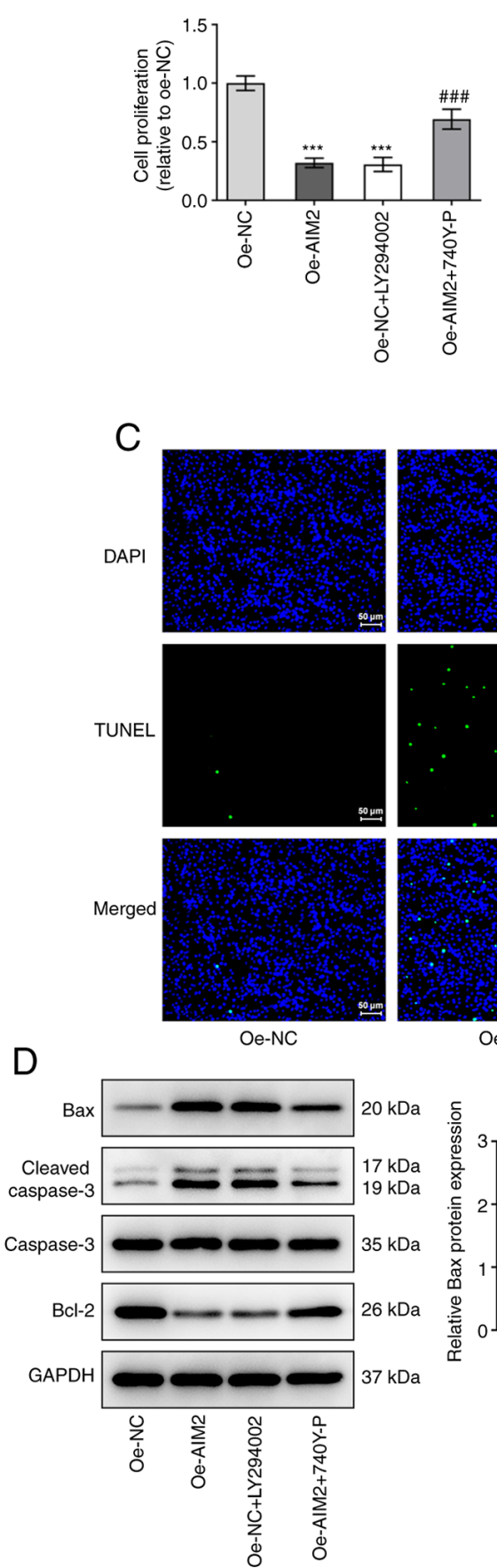

$\mathrm{B}$

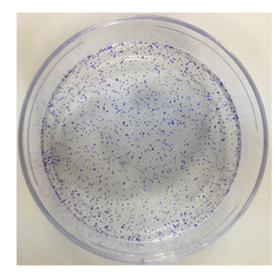

Oe-NC

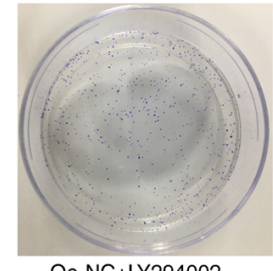

Oe-NC+LY294002

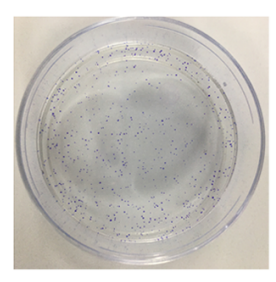

Oe-AIM2

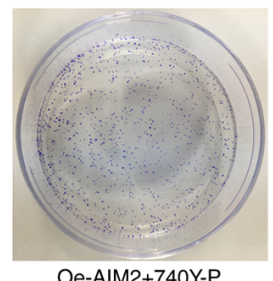

Oe-AIM2+740Y-P
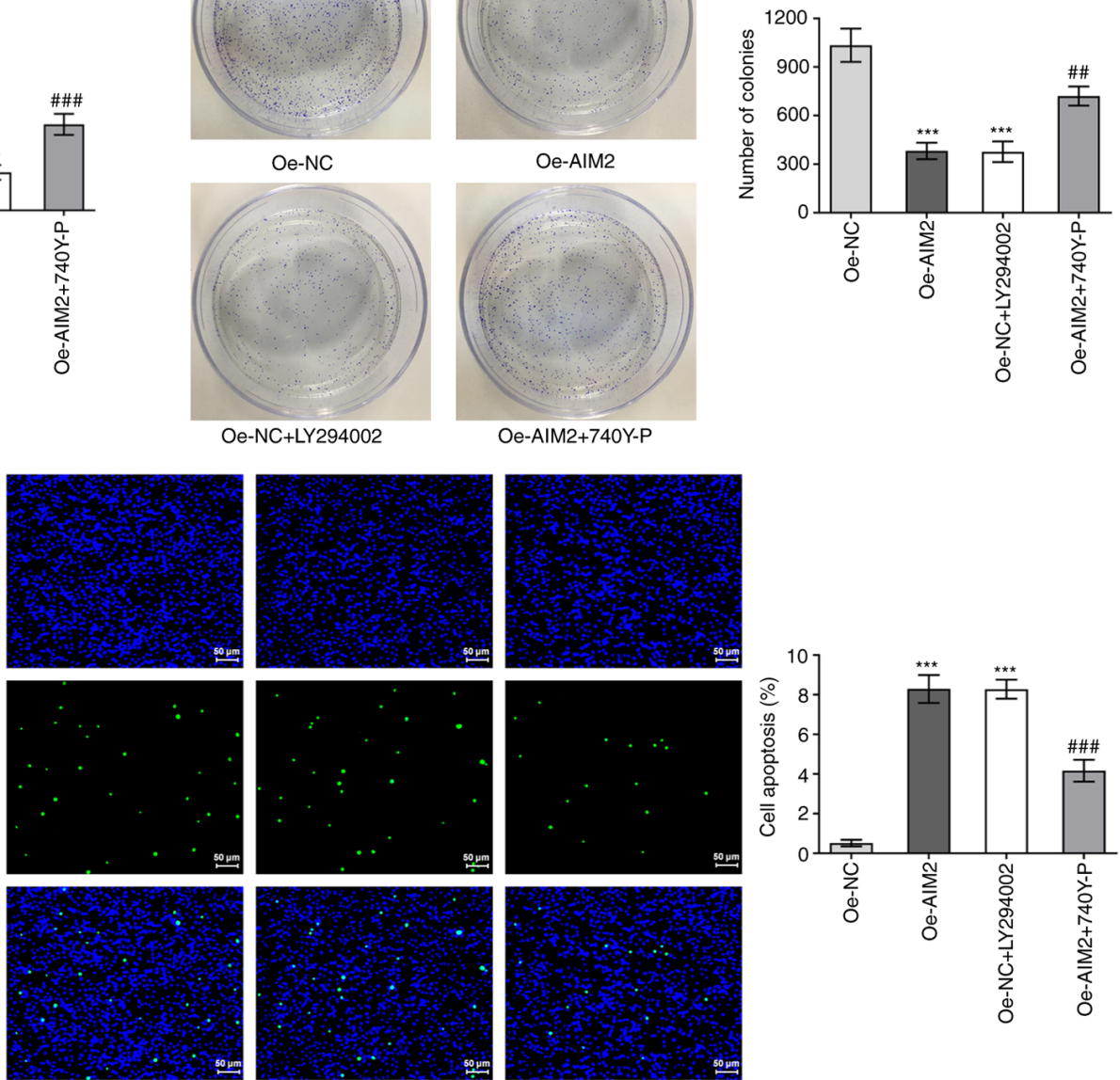

Oe-AIM2

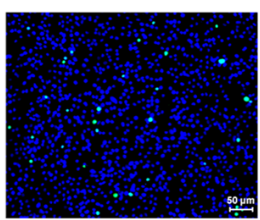

Oe-NC+LY294002

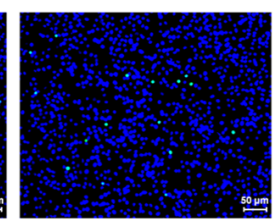

Oe-AIM2+740Y-P
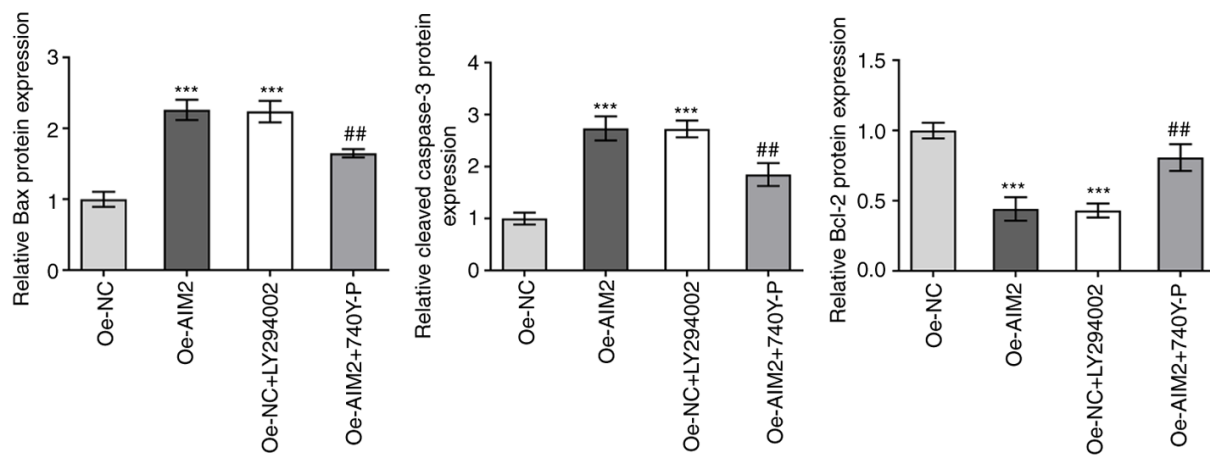

Figure 5. AIM2 overexpression inhibits the proliferation and promotes the apoptosis of osteosarcoma cells by inactivating the PI3K/AKT/mTOR signaling pathway. (A) Cell Counting Kit-8 and (B) colony formation assays were employed to detect the effect of AIM2 on the proliferation of osteosarcoma cells. (C) TUNEL staining was performed to detect apoptosis. Scale bar, $50 \mu \mathrm{m}$. (D) Detection of apoptosis-related protein expression was performed using western blotting. ${ }^{* * *} \mathrm{P}<0.001$ vs. Oe-NC; ${ }^{\# \#} \mathrm{P}<0.01,{ }^{\# \# \#} \mathrm{P}<0.001$ vs. Oe-AIM2. Experimental data are presented as the mean $\pm \mathrm{SD}$ from three independent experiments. AIM2, absent in melanoma 2; Oe-NC, empty vector plasmid; Oe-AIM2, AIM2 overexpression vector.

phosphorylates and activates the AKT protein positioned on the plasma membrane, which then activates its various downstream pathways and consequently mTOR (37). A considerable body of evidence on various cancer types has demonstrated that activation of PI3K/AKT/mTOR signaling induces a variety of malignant phenotypes, such as cell proliferation, migration and invasion $(38,39)$.
Accumulating evidence has demonstrated that the $\mathrm{PI} 3 \mathrm{~K} / \mathrm{AKT} / \mathrm{mTOR}$ signaling pathway participates in the occurrence and development of osteosarcoma, and inactivation of this signaling pathway can block the progression of osteosarcoma $(40,41)$. Previous studies have demonstrated that AIM2 regulates the activity and apoptosis of colorectal cancer cells via PI3K/AKT signaling pathways (29), and 


\section{A}

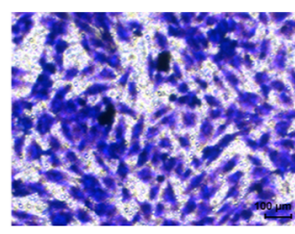

B
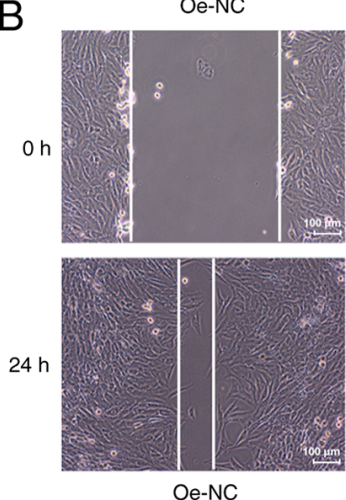

Oe-NC

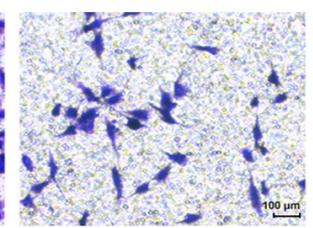

Oe-AIM2
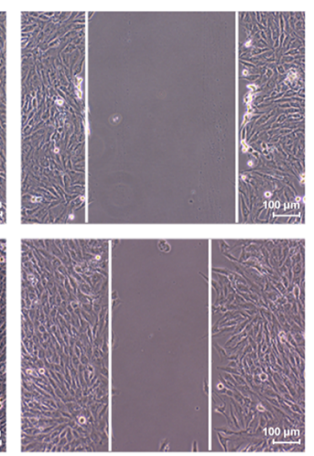

Oe-AIM2

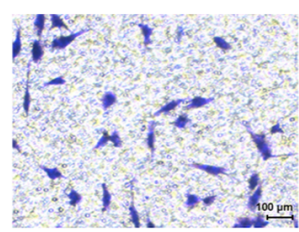

Oe-NC+LY294002
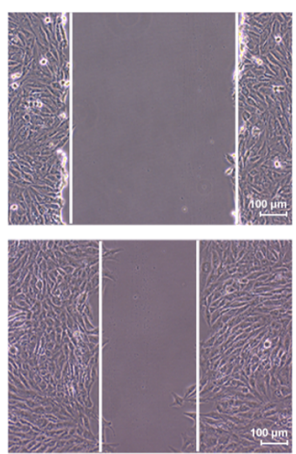

Oe-NC+LY294002

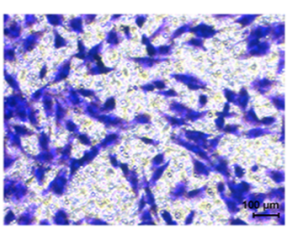

Oe-AIM2+740Y-P
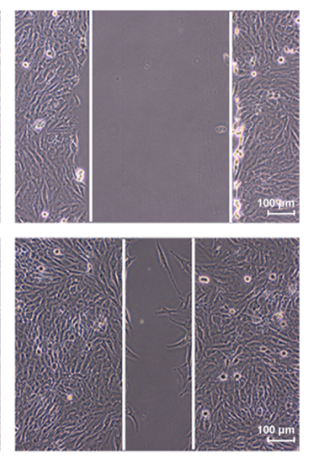

Oe-AIM2+740Y-P
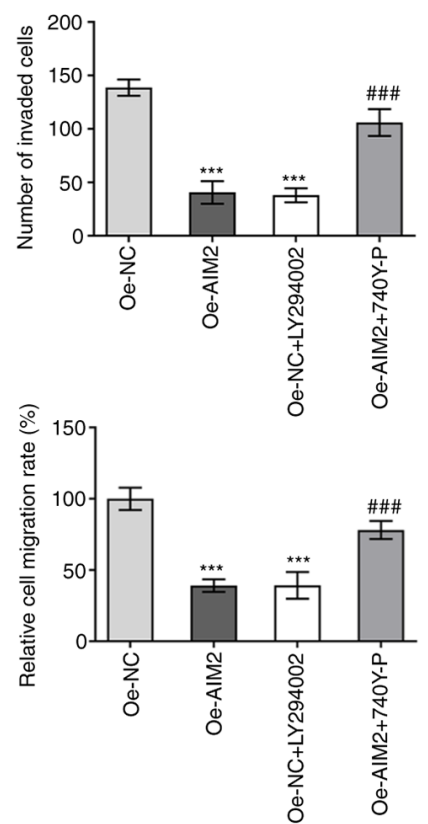

C
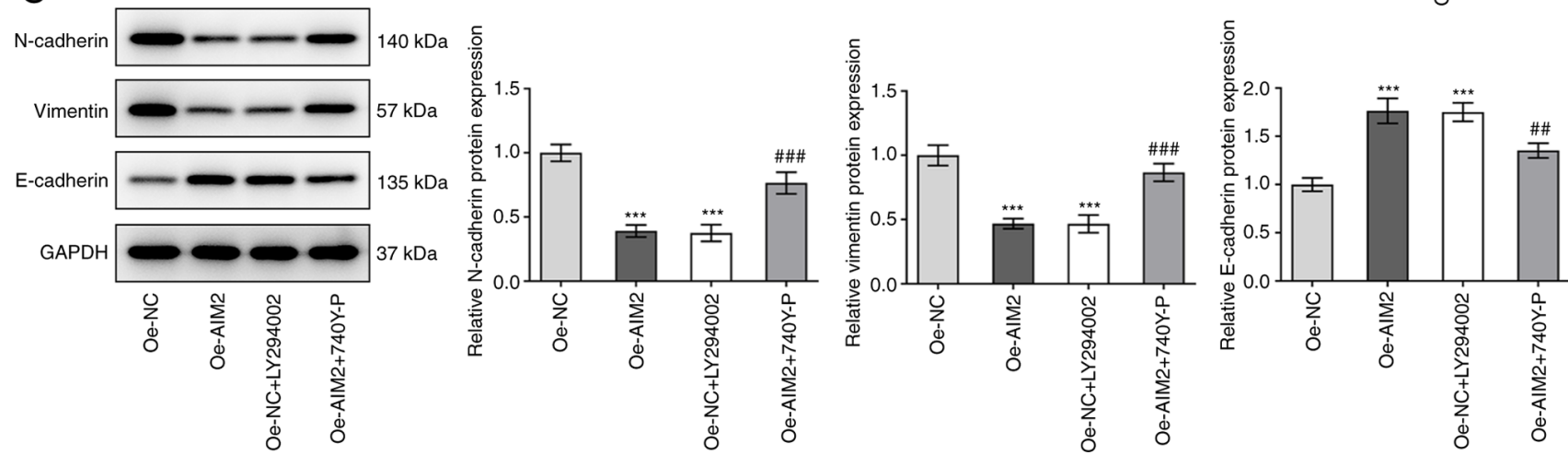

Figure 6. AIM2 overexpression inhibits the invasion, migration and EMT of osteosarcoma cells by inactivating the PI3K/AKT/mTOR signaling pathway. (A) Cell invasion was measured using a Transwell assay. Scale bar, $100 \mu \mathrm{m}$. (B) Wound healing assay was implemented to detect cell migration. Scale bar, $100 \mu \mathrm{m}$. (C) Western blotting was performed to detect EMT-related protein expression. ${ }^{* * *} \mathrm{P}<0.001$ vs. Oe-NC; ${ }^{\# \# P<0.01, ~}{ }^{\# \#} \mathrm{P}<0.001$ vs. Oe-AIM2. Experimental data are presented as the mean \pm SD from three independent experiments. EMT, epithelial-mesenchymal transition; AIM2, absent in melanoma 2; Oe-NC, empty vector plasmid; Oe-AIM2, AIM2 overexpression vector.

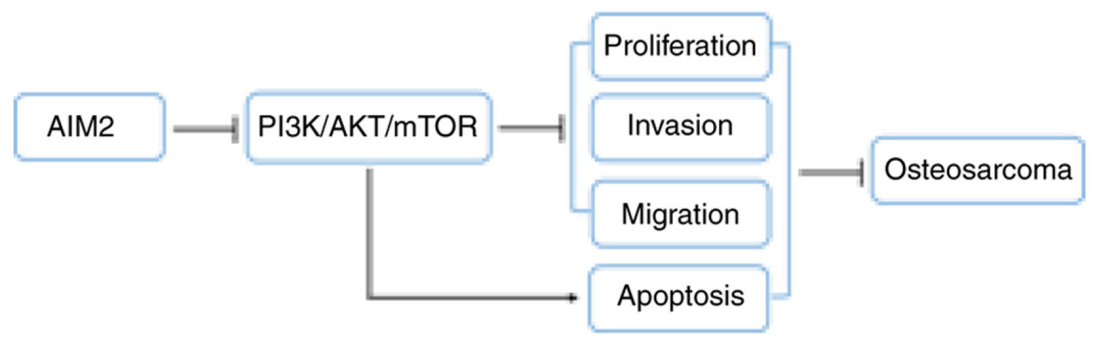

Figure 7. AIM2 inhibits the proliferation, invasion and migration, and promotes the apoptosis of osteosarcoma cells by inactivating the PI3K/AKT/mTOR signaling pathway. The schematic diagram of findings in this study. AIM2, absent in melanoma 2.

attenuates AKT phosphorylation and mTOR signaling (42). The present study revealed that AIM2 overexpression suppressed PI3K/AKT/mTOR signaling. Subsequently, to further elucidate the effect of AIM2 overexpression on the $\mathrm{PI} 3 \mathrm{~K} / \mathrm{AKT} / \mathrm{mTOR}$ signaling pathway, PI3K/AKT/mTOR pathway inhibitor LY294002 or activator 740Y-P were added. The results revealed that 740 Y-P reversed the effects of AIM2 overexpression on osteosarcoma cell proliferation, apoptosis, invasion, migration and EMT.
In conclusion, considering the present findings, it may be concluded that AIM2 can inhibit the development of osteosarcoma through inactivation of the PI3K/AKT/mTOR signaling pathway (Fig. 7). These findings suggested that AIM2 may be a promising therapeutic option for osteosarcoma and an effective approach to the targeted treatment of this disease. The lack of investigation into the upstream mechanisms of AIM2 or inclusion of animal experiments are limitations of the present study; therefore, comprehensive analysis is required in the future. 


\section{Acknowledgements}

Not applicable.

\section{Funding}

This work was supported by Natural Science Foundation of Ningxia Province (grant nos. NZ16128 and 2019AAC03193).

\section{Availability of data and materials}

The datasets used and/or analyzed during the current study are available from the corresponding author on reasonable request.

\section{Authors' contributions}

JZ and CL designed the experimental study and analyzed the data. JS performed the experiments. KW and XW drafted the manuscript and interpreted the data. KW and XW confirm the authenticity of all the raw data. All authors have read and approved the final manuscript.

\section{Ethics approval and consent to participate}

Not applicable.

\section{Patient consent for publication}

Not applicable.

\section{Competing interests}

The authors declare that they have no competing interests.

\section{References}

1. Raymond AK and Jaffe N: Osteosarcoma multidisciplinary approach to the management from the pathologist's perspective. Cancer Treat Res 152: 63-84, 2009.

2. Cortini M, Avnet S and Baldini N: Mesenchymal stroma: Role in osteosarcoma progression. Cancer Lett 405: 90-99, 2017.

3. Luetke A, Meyers PA, Lewis I and Juergens H: Osteosarcoma treatment-where do we stand? A state of the art review. Cancer Treat Rev 40: 523-532, 2014.

4. Lin H, Zheng X, Lu T, Gu Y, Zheng C and Yan H: The proliferation and invasion of osteosarcoma are inhibited by miR-101 via targetting ZEB2. Biosci Rep: Feb 8, 2019 (Epub ahead of print). doi: 10.1042/BSR20181283.

5. Bielack SS, Kempf-Bielack B, Delling G, Exner GU, Flege S, Helmke K, Kotz R, Salzer-Kuntschik M, Werner M, Winkelmann W, et al: Prognostic factors in high-grade osteosarcoma of the extremities or trunk: An analysis of 1,702 patients treated on neoadjuvant cooperative osteosarcoma study group protocols. J Clin Oncol 20: 776-790, 2002.

6. Moore DD and Luu HH: Osteosarcoma. Cancer Treat Res 162: 65-92, 2014

7. Lugrin J and Martinon F: The AIM2 inflammasome: Sensor of pathogens and cellular perturbations. Immunol Rev 281: 99-114, 2018.

8. Komada T, Chung H, Lau A, Platnich JM, Beck PL, Benediktsson H, Duff HJ, Jenne CN and Muruve DA: Macrophage uptake of necrotic cell DNA activates the AIM2 inflammasome to regulate a proinflammatory phenotype in CKD. J Am Soc Nephrol 29: 1165-1181, 2018.

9. Zhao ZZ, Zheng XL and Jiang ZS: Emerging roles of absent in melanoma 2 in cardiovascular diseases. Clin Chim Acta 511: 14-23, 2020.
10. Wilson JE, Petrucelli AS, Chen L, Koblansky AA, Truax AD, Oyama Y, Rogers AB, Brickey WJ, Wang Y, Schneider M, et al: Inflammasome-independent role of AIM2 in suppressing colon tumorigenesis via DNA-PK and Akt. Nat Med 21: 906-913, 2015.

11. Ma X, Guo P, Qiu Y, Mu K, Zhu L, Zhao W, Li T and Han L: Loss of AIM2 expression promotes hepatocarcinoma progression through activation of mTOR-S6K1 pathway. Oncotarget 7: 36185-36197, 2016.

12. PonomarevaL,Liu H,Duan X,Dickerson E, Shen H,Panchanathan R and Choubey D: AIM2, an IFN-inducible cytosolic DNA sensor, in the development of benign prostate hyperplasia and prostate cancer. Mol Cancer Res 11: 1193-1202, 2013.

13. Chen IF, Ou-Yang F, Hung JY, Liu JC, Wang H, Wang SC, Hou MF, Hortobagyi GN and Hung MC: AIM2 suppresses human breast cancer cell proliferation in vitro and mammary tumor growth in a mouse model. Mol Cancer Ther 5: 1-7, 2006.

14. Kondo Y, Nagai K, Nakahata S, Saito Y, Ichikawa T, Suekane A, Taki T, Iwakawa R, Enari M, Taniwaki M, et al: Overexpression of the DNA sensor proteins, absent in melanoma 2 and interferon-inducible 16 , contributes to tumorigenesis of oral squamous cell carcinoma with p53 inactivation. Cancer Sci 103: 782-790, 2012.

15. Farshchian M, Nissinen L, Siljamaki E, Riihilä P, Piipponen M, Kivisaari A, Kallajoki M, Grénman R, Peltonen J, Peltonen S, et al: Tumor cell-specific AIM2 regulates growth and invasion of cutaneous squamous cell carcinoma. Oncotarget 8: 45825-45836, 2017.

16. Zhang M, Jin C, Yang Y, Wang K, Zhou Y, Zhou Y, Wang R, $\mathrm{Li} \mathrm{T}$ and $\mathrm{Hu}$ R: AIM2 promotes non-small-cell lung cancer cell growth through inflammasome-dependent pathway. J Cell Physiol 234: 20161-20173, 2019.

17. Hu K, Dai HB and Qiu ZL: mTOR signaling in osteosarcoma: Oncogenesis and therapeutic aspects (Review). Oncol Rep 36: 1219-1225, 2016

18. Xu M, Wang J, Li H, Zhang Z and Cheng Z: AIM2 inhibits colorectal cancer cell proliferation and migration through suppression of Gli1. Aging (Albany NY) 13: 1017-1031, 2020.

19. Zhang Y, Weng Q, Han J and Chen J: Alantolactone suppresses human osteosarcoma through the PI3K/AKT signaling pathway. Mol Med Rep 21: 675-684, 2020.

20. Livak KJ and Schmittgen TD: Analysis of relative gene expression data using real-time quantitative PCR and the 2(-Delta Delta C(T)) method. Methods 25: 402-408, 2001.

21. Sharma BR, Karki R and Kanneganti TD: Role of AIM2 inflammasome in inflammatory diseases, cancer and infection. Eur J Immunol 49: 1998-2011, 2019.

22. Li Y, Wang W, Li A, Huang W, Chen S, Han F and Wang L: Dihydroartemisinin induces pyroptosis by promoting the AIM2/caspase-3/DFNA5 axis in breast cancer cells. Chem Biol Interact 340: 109434, 2021.

23. Wang B, Tian Y and Yin Q: AIM2 inflammasome assembly and signaling. Adv Exp Med Biol 1172: 143-155, 2019.

24. Jin H, Jin X, Cao B and Wang W: Berberine affects osteosarcoma via downregulating the caspase-1/IL-1 $\beta$ signaling axis. Oncol Rep 37: 729-736, 2017.

25. Sun Y and Guo Y: Expression of Caspase-1 in breast cancer tissues and its effects on cell proliferation, apoptosis and invasion. Oncol Lett 15: 6431-6435, 2018.

26. Huang TH, Zhang P, Li W, Zhao T, Zhang Z, Chen S, Yang Y, Feng Y, Li F, Shirley Liu X, et al: G9A promotes tumor cell growth and invasion by silencing CASP1 in non-small-cell lung cancer cells. Cell Death Dis 8: e2726, 2017.

27. Seyfried TN and Huysentruyt LC: On the origin of cancer metastasis. Crit Rev Oncog 18: 43-73, 2013.

28. Avanzini S and Antal T: Cancer recurrence times from a branching process model. PLoS Comput Biol 15: e1007423, 2019.

29. Patsos G, Germann A, Gebert J and Dihlmann S: Restoration of absent in melanoma 2 (AIM2) induces G2/M cell cycle arrest and promotes invasion of colorectal cancer cells. Int J Cancer 126: 1838-1849, 2010

30. Man SM, Zhu Q, Zhu L, Liu Z, Karki R, Malik A, Sharma D, Li L, Malireddi RK, Gurung P, et al: Critical Role for the DNA Sensor AIM2 in stem cell proliferation and cancer. Cell 162: 45-58, 2015.

31. Chen J, Wang Z and Yu S: AIM2 regulates viability and apoptosis in human colorectal cancer cells via the PI3K/Akt pathway. Onco Targets Ther 10: 811-817, 2017.

32. Poh L, Fann DY, Wong P, Lim HM, Foo SL, Kang SW, Rajeev V, Selvaraji S, Iyer VR, Parathy N, et al: AIM2 inflammasome mediates hallmark neuropathological alterations and cognitive impairment in a mouse model of vascular dementia. Mol Psychiatry 26: 4544-4560, 2021. 
33. Chai D, Shan H, Wang G, Li H, Fang L, Song J, Zhang Q, Bai J and Zheng J: AIM2 is a potential therapeutic target in human renal carcinoma and suppresses its invasion and metastasis via enhancing autophagy induction. Exp Cell Res 370: 561-570, 2018.

34. Chen SL, Liu LL, Lu SX, Luo RZ, Wang CH, Wang H, Cai SH, Yang X, Xie D, Zhang CZ and Yun JP: HBx-mediated decrease of AIM2 contributes to hepatocellular carcinoma metastasis. Mol Oncol 11: 1225-1240, 2017.

35. Xia $\mathrm{P}$ and $\mathrm{Xu} \mathrm{XY}$ : $\mathrm{PI} 3 \mathrm{~K} / \mathrm{Akt} / \mathrm{mTOR}$ signaling pathway in cancer stem cells: From basic research to clinical application. Am J Cancer Res 5: 1602-1609, 2015.

36. Zhang J, Yu XH, Yan YG, Wang $\mathrm{C}$ and Wang WJ: PI3K/Akt signaling in osteosarcoma. Clin Chim Acta 444: 182-192, 2015.

37. Ersahin T, Tuncbag N and Cetin-Atalay R: The PI3K/AKT/mTOR interactive pathway. Mol Biosyst 11: 1946-1954, 2015.

38. Tang H, Zhu D, Zhang G, Luo X and Xie W: AFAP1-AS1 promotes proliferation of pituitary adenoma cells through miR-103a-3p to Activate PI3K/AKT signaling pathway. World Neurosurg 130: e888-e898, 2019.

39. Rodrigues Alves AP, Fernandes JC, Fenerich BA, CoelhoSilva JL, Scheucher PS, Simões BP, Rego EM, Ridley AJ, Machado-Neto JA and Traina F: IGF1R/IRS1 targeting has cytotoxic activity and inhibits PI3K/AKT/mTOR and MAPK signaling in acute lymphoblastic leukemia cells. Cancer Lett 456: $59-68,2019$.
40. Wang B and Li J: Piceatannol suppresses the proliferation and induced apoptosis of osteosarcoma cells through PI3K/AKT/mTOR pathway. Cancer Manag Res 12: 2631-2640, 2020.

41. Jin R, Jin YY, Tang YL, Yang HJ, Zhou XQ and Lei Z: GPNMB silencing suppresses the proliferation and metastasis of osteosarcoma cells by blocking the PI3K/Akt/mTOR signaling pathway. Oncol Rep 39: 3034-3040, 2018.

42. Chou WC, Guo Z, Guo H, Chen L, Zhang G, Liang K, Xie L, Tan X, Gibson SA, Rampanelli E, et al: AIM2 in regulatory T cells restrains autoimmune diseases. Nature 591: 300-305, 2021.

(i) (5) $\ominus$ This work is licensed under a Creative Commons Attribution-NonCommercial-NoDerivatives 4.0 International (CC BY-NC-ND 4.0) License. 